An International Journal of Optimization and Control: Theories \& Applications

ISSN: 2146-0957 eISSN: 2146-5703

Vol.7, No.3, pp.275-280 (2017)

https://doi.org/10.11121/ijocta.01.2017.00493

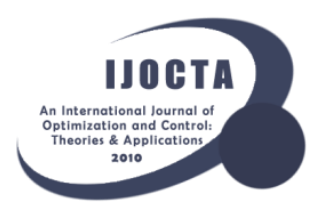

RESEARCH ARTICLE

\title{
Analytical solutions of Phi-four equation
}

\author{
Seyma Tuluce Demiray *, Hasan Bulut \\ Department of Mathematics, Firat University, Turkey \\ seymatuluce@gmail.com,hbulut@firat.edu.tr
}

\begin{tabular}{l} 
ARTICLE INFO \\
\hline Article history: \\
Received: 16 June 2017 \\
Accepted: 16 September 2017 \\
Available Online: 7 November 2017 \\
\hline Keywords: \\
Phi-four equation \\
Modified exp $(-\Omega(\xi))$-expansion function \\
method \\
Dark soliton solutions \\
Trigonometric function solution \\
Mathematica 9
\end{tabular}

\section{ABSTRACT}

This study bases attention on new analytical solutions of Phi-four equation. The modified $\exp (-\Omega(\xi))$-expansion function method (MEFM) has been used to obtain analytical solutions of the Phi-four equation. By using this method, dark soliton solutions and trigonometric function solution of the Phi-four equation have been found.

AMS Classification 2010:

35-04, 35C08, 35N05, 68N15

\section{Introduction}

Nonlinear evolution equations (NLEEs) are considerably used to identify a variety of physical circumstances in the areas such as quantum field theory, hydrodynamics, chemical kinematics, geochemistry, electricity, elastic media and plasma physics.

Recently, many researchers have introduced a lot of methods to acquire exact solutions of NLEEs such as G'/G-expansion method [1], modified extended tanhfunction method [2], sine-cosine method [3], expfunction method [4], modified simple equation method [4], extended trial equation method [5], generalized Kudryashov method [6] . In this study, MEFM [7] will be implemented to find new analytical solutions of Phi-four equation.

We consider Phi-four equation [8-11],

$$
u_{t t}-a u_{x x}-u+u^{3}=0, a>0,
$$

where $a$ is real constant. This equation can be investigated as a special form of the Klein-Gordon equation that patterns the phenomenon in particle physics where kink and anti-kink solitary waves interact [12].

Many scientists have used exact and numerical solutions of Phi-four equation to research some methods such as the sine-cosine method [8], the auxiliary equation method [9], the modified simple equation method [10], homotopy perturbation method [11], homotopy analysis method [11] and Adomian decomposition method [11].

In this article, the basic interest is to construct new exact solutions of Phi-four equation via MEFM. In Sec. 2, we clarify basic facts of MEFM. In Sec. 3, we find new exact solutions of the Phi-four equation via MEFM.

\section{Basic facts of method}

The fundamental properties of MEFM are introduced in this section. MEFM is predicated on the exp $(-\Omega(\xi))$-expansion function method [13-16]. In order to implement this method to the nonlinear partial differential equations, we handle it as follows:

$$
P\left(u, u_{t}, u_{x}, u_{t t}, u_{x x}, \cdots\right)=0
$$

where $u=u(x, t)$ is an unknown function, $P$ is a polynomial in $u(x, t)$ and its derivatives, in which the highest order derivatives and nonlinear terms are included and the subscripts demonstrate the partial derivatives. The fundamental stages of the method are defined as follows:

Step 1: Let us investigate the following traveling transformation identified by

${ }^{*}$ Corresponding author 


$$
u(x, t)=U(\xi), \quad \xi=k(x-c t) .
$$

Using Eq. (3), we can turn Eq. (2) into a nonlinear ordinary differential equation (NODE) described by:

$$
\operatorname{NODE}\left(U, U^{\prime}, U^{\prime \prime}, U^{\prime \prime \prime}, \cdots\right)=0
$$

where NODE is a polynomial of $U$ and its derivatives and the superscripts demonstrate the ordinary derivatives according to $\xi$.

Step 2: Assume the traveling wave solution of Eq. (4) can be shown as follows:

$$
\begin{aligned}
& U(\xi)=\frac{\sum_{i=0}^{N} A_{i}[\exp (-\Omega(\xi))]^{i}}{\sum_{j=0}^{M} B_{j}[\exp (-\Omega(\xi))]^{j}} \\
& =\frac{A_{0}+A_{1} \exp (-\Omega)+\cdots+A_{N} \exp (\mathrm{N}(-\Omega))}{B_{0}+B_{1} \exp (-\Omega)+\cdots+B_{M} \exp (\mathrm{M}(-\Omega))},
\end{aligned}
$$

where $A_{i}, B_{j},(0 \leq i \leq N, 0 \leq j \leq M)$ are constants to be described later, such that $A_{N} \neq 0, B_{M} \neq 0$, and $\Omega=\Omega(\xi)$ is solution of the following ordinary differential equation:

$$
\Omega^{\prime}(\xi)=\exp (-\Omega(\xi))+\mu \exp (\Omega(\xi))+\lambda .
$$

There are the following solution families of Eq. (6):

Familyl: When $\mu \neq 0, \lambda^{2}-4 \mu>0$,

$$
\Omega(\xi)=\ln \left(\frac{-\sqrt{\lambda^{2}-4 \mu}}{2 \mu} \tanh \left(\frac{\sqrt{\lambda^{2}-4 \mu}}{2}(\xi+E)\right)-\frac{\lambda}{2 \mu}\right) .
$$

Family2: When $\mu \neq 0, \lambda^{2}-4 \mu<0$,

$\Omega(\xi)=\ln \left(\frac{\sqrt{-\lambda^{2}+4 \mu}}{2 \mu} \tan \left(\frac{\sqrt{-\lambda^{2}+4 \mu}}{2}(\xi+E)\right)-\frac{\lambda}{2 \mu}\right)$.

Family3: When $\mu=0, \lambda \neq 0$, and $\lambda^{2}-4 \mu>0$,

$$
\Omega(\xi)=-\ln \left(\frac{\lambda}{\exp (\lambda(\xi+E))-1}\right) .
$$

Family4: When $\mu \neq 0, \lambda \neq 0$, and $\lambda^{2}-4 \mu=0$,

$$
\Omega(\xi)=\ln \left(-\frac{2 \lambda(\xi+E)+4}{\lambda^{2}(\xi+E)}\right) .
$$

Family5: When $\mu=0, \lambda=0$, and $\lambda^{2}-4 \mu=0$,

$$
\Omega(\xi)=\ln (\xi+E) \text {. }
$$

such that $A_{0}, A_{1}, A_{2}, \cdots A_{N}, B_{0}, B_{1}, B_{2}, \cdots B_{M}, E, \lambda, \mu$ are constants to be described later. The positive integers $N$ and $M$ can be identified by taking into consideration the homogeneous balance between the highest order derivatives and the nonlinear terms arising in Eq. (5).

Step 3: Embedding Eqs. (6) and (7-11) into Eq. (5), we attain a polynomial of $\exp (-\Omega(\xi))$. We compensate all the coefficients of same power of $\exp (-\Omega(\xi))$ to zero. This process provides a system of equations which can be solved to obtain $A_{0}, A_{1}, A_{2}, \cdots A_{N}, B_{0}, B_{1}, B_{2}, \cdots B_{M}, E, \lambda, \mu \quad$ by using Wolfram Mathematica 9. Putting the values of $A_{0}, A_{1}, A_{2}, \cdots A_{N}, B_{0}, B_{1}, B_{2}, \cdots B_{M}, E, \lambda, \mu$ into Eq. (5), the general solutions of Eq. (5) fulfil the determination of the solution of Eq. (1).

\section{MEFM for Phi-Four Equation}

In this section, we look for the exact solutions of Eq. (1) by using MEFM.

We find the travelling wave solutions of Eq. (1) by using the wave variables

$$
u(x, t)=u(\xi), \xi=k(x-c t),
$$

where $k$ and $c$ are arbitrary constants.

Putting Eq. (13) into Eq. (1),

$$
u_{t t}=k^{2} c^{2} u^{\prime \prime}, u_{x x}=k^{2} u^{\prime \prime}
$$

we obtain following equation

$$
k^{2}\left(c^{2}-a\right) u^{\prime \prime}-u+u^{3}=0,
$$

where the prime indicates the derivative with regard to $\xi$.

Using balance principle in Eq. (14), we obtain

$$
N=M+1 \text {. }
$$

If we take $M=1$ so $N=2$, we can acquire

$U=\frac{A_{0}+A_{1} \exp (-\Omega)+A_{2} \exp (2(-\Omega))}{B_{0}+B_{1} \exp (-\Omega)}=\frac{\Upsilon}{\Psi}$,

and

$$
\begin{gathered}
U^{\prime}=\frac{\Upsilon^{\prime} \Psi-\Psi^{\prime} \Upsilon}{\Psi^{2}} \\
U^{\prime \prime}=\frac{\Upsilon^{\prime \prime} \Psi^{3}-\Psi^{2} \Upsilon^{\prime} \Psi^{\prime}-\left(\Psi^{\prime \prime} \Upsilon+\Psi^{\prime} \Upsilon^{\prime}\right) \Psi^{2}+2\left(\Psi^{\prime}\right)^{2} \Upsilon \Psi}{\Psi^{4}} \\
\vdots
\end{gathered}
$$

where $A_{2} \neq 0$ and $B_{1} \neq 0$. When we use Eq.(16) and Eq.(18) in Eq.(14) we get a system of algebraic equations from the coefficients of polynomial of $\exp (-\Omega(\xi))$. By solving this system of algebraic equations by using Wolfram Mathematica 9, it yields us the following coefficients: 


\section{Case 1:}

$A_{0}=\frac{\lambda B_{0}}{\sqrt{\lambda^{2}-4 \mu}}, A_{1}=\frac{2 B_{0}+\lambda B_{1}}{\sqrt{\lambda^{2}-4 \mu}}$,

$A_{2}=\frac{2 B_{1}}{\sqrt{\lambda^{2}-4 \mu}}, c=-\frac{\sqrt{-2+a k^{2}\left(\lambda^{2}-4 \mu\right)}}{\sqrt{k^{2}\left(\lambda^{2}-4 \mu\right)}}$,

$B_{0}=B_{0}, B_{1}=B_{1}, \lambda=\lambda, \mu=\mu$.

\section{Case 2:}

$A_{0}=\frac{A_{1} B_{0}}{2 B_{1}}, A_{1}=A_{1}, A_{2}=\frac{A_{1} B_{1}}{2 B_{0}}$,

$\lambda=\frac{2 B_{0}}{B_{1}}, \mu=B_{0}^{2}\left(-\frac{4}{A_{1}^{2}}+\frac{1}{B_{1}^{2}}\right)$,

$c=-\frac{\sqrt{-\frac{A_{1}^{2}}{2}+4 a k^{2} B_{0}^{2}}}{2 k B_{0}}, B_{0}=B_{0}, B_{1}=B_{1}$.

\section{Case 3:}

$A_{0}=\frac{B_{0}\left(A_{1}^{2}+B_{1}^{2}\right)}{2 A_{1} B_{1}}, A_{2}=\frac{A_{1} B_{1}}{2 B_{0}}$,

$\lambda=\frac{2 B_{0}}{B_{1}}, \mu=B_{0}^{2}\left(-\frac{1}{A_{1}^{2}}+\frac{1}{B_{1}^{2}}\right)$,

$c=-\frac{\sqrt{-\frac{A_{1}^{2}}{2}+4 a k^{2} B_{0}^{2}}}{2 k B_{0}}, A_{1}=A_{1}$,

$B_{0}=B_{0}, B_{1}=B_{1}$.

\section{Case 4:}

$A_{0}=\frac{A_{1} B_{0}}{2 B_{1}}+\frac{B_{0} B_{1}}{A_{1}}, A_{2}=\frac{A_{1} B_{1}}{2 B_{0}}$,

$\lambda=\frac{2 B_{0}}{B_{1}}, \mu=B_{0}^{2}\left(\frac{2}{A_{1}^{2}}+\frac{1}{B_{1}^{2}}\right)$,

$c=-\frac{\sqrt{-\frac{A_{1}^{2}}{2}+4 a k^{2} B_{0}^{2}}}{2 k B_{0}}, A_{1}=A_{1}$,

$B_{0}=B_{0}, B_{1}=B_{1}$.

Embedding Eq. (19) together with Eqs. (3) and (7) in Eq. (16), we obtain dark soliton solution for Eq. (1) as follows:

$u_{1}(x, t)=\frac{\lambda^{2}-4 \mu+\lambda \sqrt{\lambda^{2}-4 \mu} \tanh [f(x, t)]}{\sqrt{\lambda^{2}-4 \mu}\left(\lambda+\sqrt{\lambda^{2}-4 \mu} \tanh [f(x, t)]\right)}$, where

$$
f(x, t)=\frac{1}{2} \sqrt{\lambda^{2}-4 \mu}\left[E+k\left(x+\frac{t \sqrt{-2+a k^{2}\left(\lambda^{2}-4 \mu\right)}}{\sqrt{k^{2}\left(\lambda^{2}-4 \mu\right)}}\right)\right],
$$

and $\mu \neq 0, \lambda^{2}-4 \mu>0$.

Substituting Eq. (20) together with Eqs. (3) and (7) in Eq. (16), we find dark soliton solution for Eq. (1) as follows:

$$
u_{2}(x, t)=\frac{A_{1} \tanh [g(x, t)]+2 B_{1}}{A_{1}+2 B_{1} \tanh [g(x, t)]},
$$

where

$g(x, t)=\frac{4(E+k x) B_{0}+\sqrt{2} t \sqrt{-A_{1}^{2}+8 a k^{2} B_{0}^{2}}}{2 A_{1}}$, and

$-4 B_{0}^{2}\left(-\frac{4}{A_{1}^{2}}+\frac{1}{B_{1}^{2}}\right)+\frac{4 B_{0}^{2}}{B_{1}^{2}}>0$.

Putting Eq. (21) together with Eqs. (3) and (7) in Eq. (16), we find dark soliton solution for Eq. (1) as follows:

$$
\begin{aligned}
u_{3}(x, t)= & \frac{1}{2}\left[\frac{A_{1}}{B_{1}}+\frac{B_{1}}{A_{1}}+\frac{A_{1}^{2}-B_{1}^{2}}{A_{1}\left(B_{1}+A_{1} \tanh [h(x, t)]\right)}\right] \\
& +\frac{1}{2}\left[\frac{-A_{1}^{2}+B_{1}^{2}}{B_{1}\left(A_{1}+B_{1} \tanh [h(x, t)]\right)}\right]
\end{aligned}
$$

where

$$
\begin{aligned}
& h(x, t)=\frac{B_{0}}{A_{1}}\left(E+k x+t \frac{\sqrt{-\frac{A_{1}^{2}}{2}+4 a k^{2} B_{0}^{2}}}{2 B_{0}}\right), \\
& \text { and }-4 B_{0}^{2}\left(-\frac{1}{A_{1}^{2}}+\frac{1}{B_{1}^{2}}\right)+\frac{4 B_{0}^{2}}{B_{1}^{2}}>0 .
\end{aligned}
$$

Embedding Eq. (22) together with Eqs. (3) and (7) in Eq. (16), we obtain trigonometric function solution for Eq. (1) as follows:

$$
u_{4}(x, t)=\frac{\left(A_{1}^{2}+2 B_{1}^{2}\right) \sec ^{2}[k(x, t)]}{\left(2 B_{1}+\sqrt{2} A_{1} \tan [k(x, t)]\right) \cdot\left(-A_{1}+\sqrt{2} B_{1} \tan [k(x, t)]\right)},
$$

where

$k(x, t)=\frac{\sqrt{2} B_{0}}{A_{1}}\left(E+k x+t \frac{\sqrt{-\frac{A_{1}^{2}}{2}+4 a k^{2} B_{0}^{2}}}{2 B_{0}}\right)$, and $-4 B_{0}^{2}\left(\frac{2}{A_{1}^{2}}+\frac{1}{B_{1}^{2}}\right)+\frac{4 B_{0}^{2}}{B_{1}^{2}}>0$. 

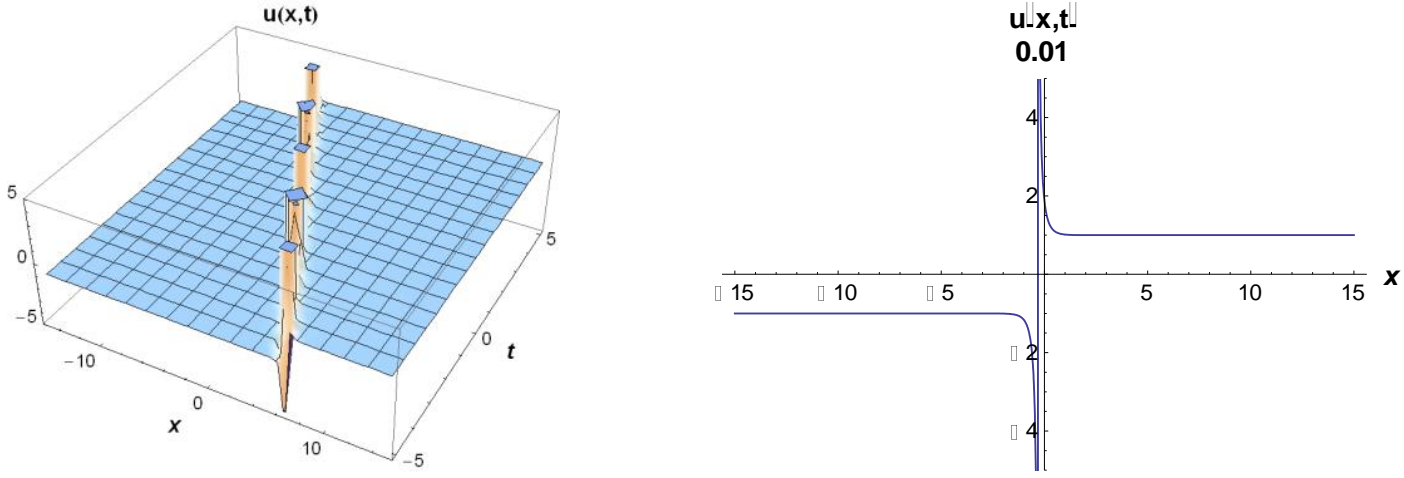

Figure 1. The 3D and 2D surfaces of Eq. (23) for $\lambda=0.3, \mu=-0.2, k=4, a=2, E=0.5,-15<x<15,-5<t<5$ and $t=0.01$ for $2 \mathrm{D}$ surface.
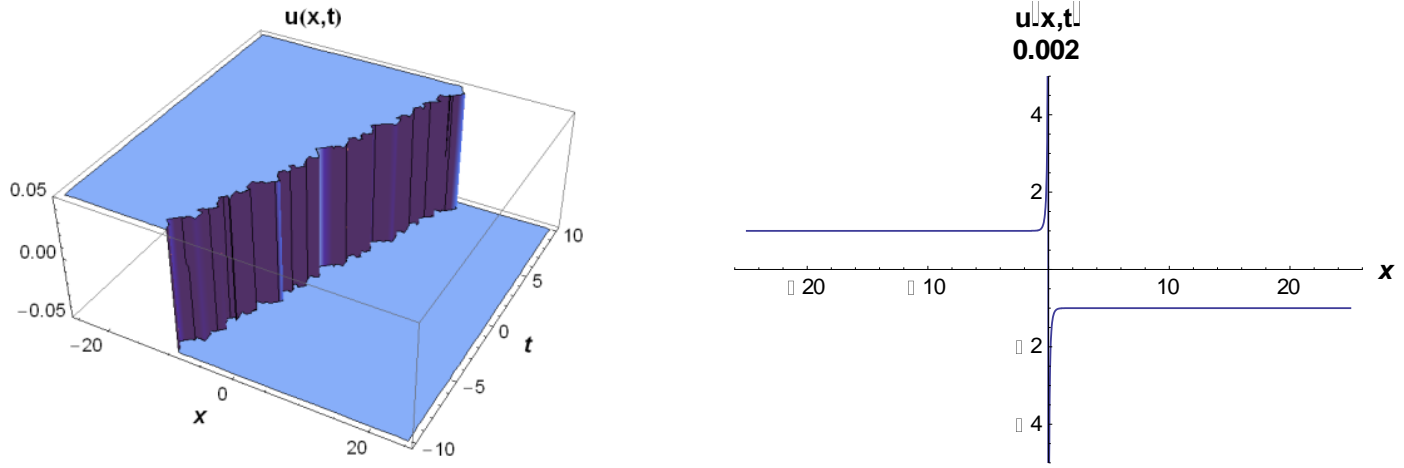

Figure 2. The 3D and 2D surfaces of Eq. (24) for $k=3, a=1, A_{1}=2, B_{0}=-1, B_{1}=4, E=0.3,-25<x<25,-10<t<10$ and $t=0.002$ for 2D surface.
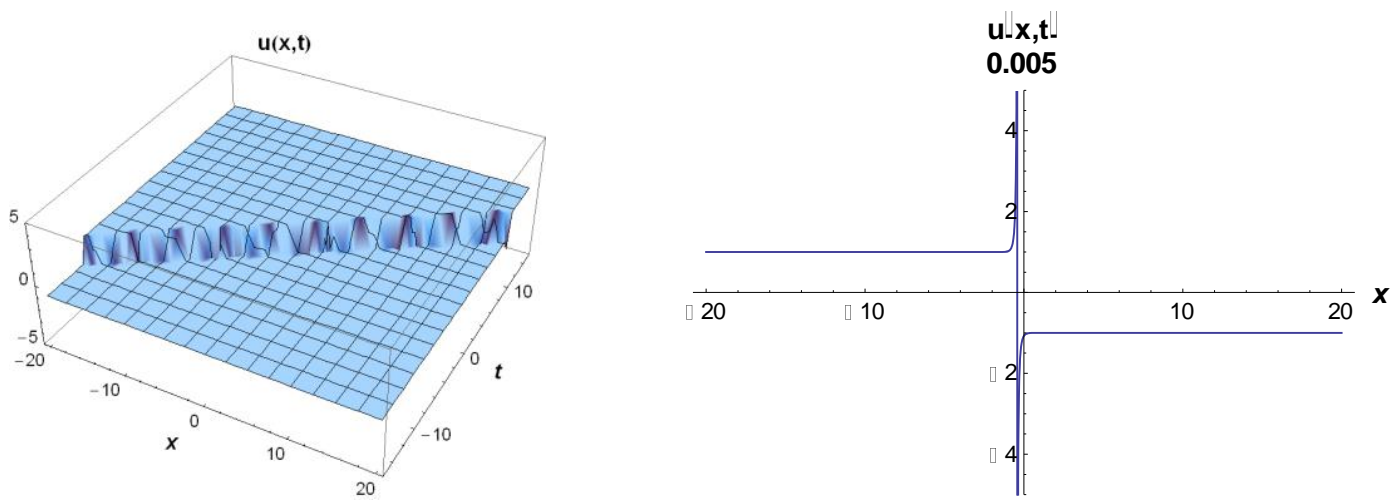

Figure 3. The 3D and 2D surfaces of Eq. (25) for $k=1, a=4, A_{1}=1, B_{0}=-2, B_{1}=3, E=0.6,-20<x<20,-15<t<15$ and $t=0.005$ for $2 \mathrm{D}$ surface. 

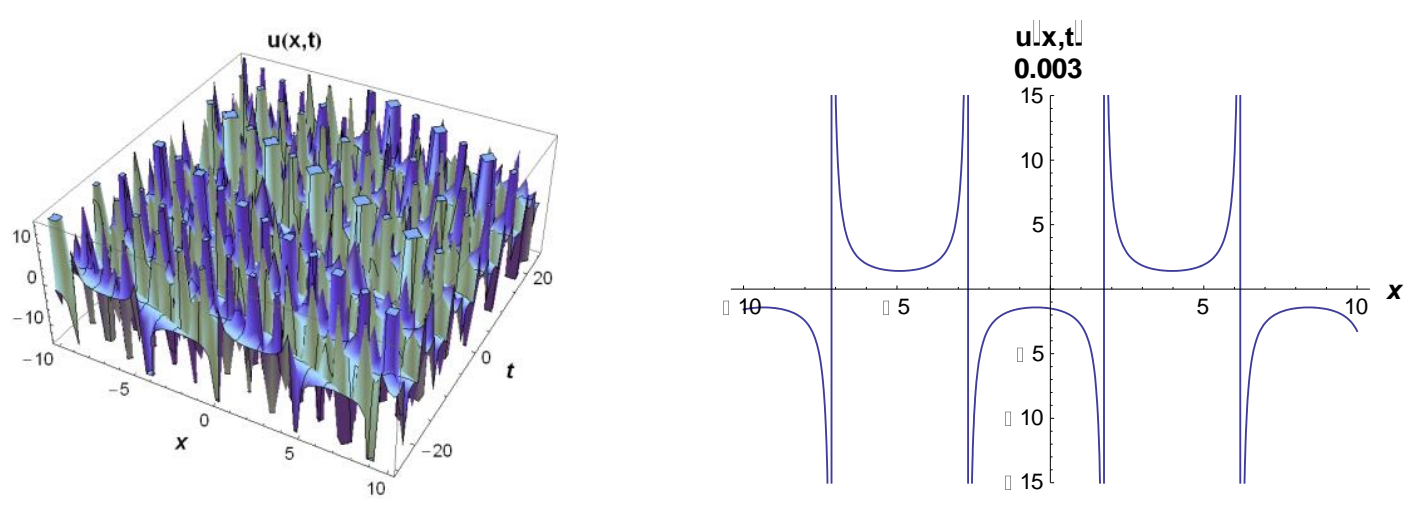

Figure 4. The 3D and 2D surfaces of Eq. (26) for $k=-1, a=3, A_{1}=4, B_{0}=-1, B_{1}=5, E=0.3,-10<x<10,-25<t<25$ and $t=0.003$ for $2 \mathrm{D}$ surface.

Remark The exact solutions of Eq. (1) were obtained via MEFM and were controlled by use of Mathematica Release 9. As far as we know, the solutions of Eq. (1) that we found in this study are new and are not indicated before.

\section{Conclusion}

In this paper, we use MEFM to find exact solutions of Phi-four equation. Then, in Figures 1-4, we plot 2D and 3D surfaces of dark soliton solutions and trigonometric function solution of Phi-four equation by using Mathematica Release 9 .

According to these data and observation, it has been deduced that this method has been influential for the exact solutions of these NLEEs and this method is highly effective and dependable in the sense that reaching analytical solutions. Thus, we can say that this method has a substantial position to attain exact solutions of NLEEs.

\section{References}

[1] Younis, M., Rizvi, S.T.R. (2015). Dispersive dark optical soliton in $(2+1)$-dimensions by $\mathrm{G}^{\prime} / \mathrm{G}$ expansion with dual-power law nonlinearity. Optik, 126, 5812-5814.

[2] Zahran, E.H.M., Khater, M.M.A. (2016). Modified extended tanh-function method and its applications to the Bogoyavlenskii equation. Applied Mathematical Modelling, 40, 17691775.

[3] Bibi, S., Mohyud-Din, S.T. (2014). Traveling wave solutions of $\mathrm{KdVs}$ using sine-cosine method. Journal of the Association of Arab Universities for Basic and Applied Sciences, 15, 90-93.

[4] Khan, K., Akbar, M.A. (2014). Traveling wave solutions of the $(2+1)$-dimensional Zoomeron equation and the Burgers equations via the MSE method and the Exp-function method. Ain Shams Engineering Journal, 5, 247-256.
[5] Tuluce Demiray, S., Bulut, H. (2015). Some Exact Solutions of Generalized Zakharov System. Waves in Random and Complex Media, 25(1), 75-90.

[6] Tuluce Demiray, S., Bulut, H. (2017). New soliton solutions of Davey-Stewartson equation with power-law nonlinearity. Opt Quant Electron, 49(117), 1-8.

[7] Baskonus, H. M., Bulut, H., Atangana, A. (2016). On the complex and hyperbolic structures of the longitudinal wave equation in a magneto-electro-elastic circular rod. Smart Materials and Structures, 25(3), 1-8.

[8] Wazwaz, A.M. (2004). A sine-cosine method for handling nonlinear wave equations. Mathematical and Computer Modelling, 40, 499508.

[9] Bekir, A. (2008). New Exact Travelling Wave Solutions for Regularized Long-wave, Phi-Four and Drinfeld-Sokolov Equations. International Journal of Nonlinear Science, 6(1), 46-52.

[10] Younis, M., Zafar, A. (2013). The modified simple equation method for solving nonlinear Phi-Four equation. International Journal of Innovation and Applied Studies, 2(4), 661-664.

[11] Ehsani, F., Ehsani, F., Hadi, A., Hadi, N. (2013). Analytical Solution of Phi-Four Equation. Technical Journal of Engineering and Applied Sciences, 3(14), 1378-1388.

[12] Dashen, R.F., Hasslacher, B., Neveu, A. (1975) Particle spectrum in model field theories from semi-classical functional integral technique. Physical Review D, 11, 3424-3450.

[13] Alam, M.N., Hafez, M.G., Akbar, M.A., Roshid, H.O. (2015). Exact Solutions to the $(2+1)$ Dimensional Boussinesq Equation via $\exp (\Phi(\eta))$-Expansion Method. Journal of Scientific Research, 7(3), 1-10. 
[14] Roshid, H.O., Rahman, Md. A. (2014). The $\exp (-\Phi(\eta))$-expansion method with application in the $(1+1)$-dimensional classical Boussinesq equations. Results in Physics, 4, 150-155.

[15] Abdelrahman, M.A.E., Zahran, E.H.M., Khater, M.M.A. (2015). The $\exp (-\phi(\xi))$-Expansion Method and Its Application for Solving Nonlinear Evolution Equations. International Journal of Modern Nonlinear Theory and Application, 4, 37-47.

[16] Hafez, M.G., Alam, M.N., Akbar, M.A. (2014). Application of the $\exp (-\Phi(\eta))$-expansion Method to Find Exact Solutions for the Solitary Wave Equation in an Unmagnatized Dusty Plasma. World Applied Sciences Journal, 32(10), 2150-2155.
Seyma Tuluce Demiray is an Assoc. Prof. Dr. in Department of Mathematics at Firat University; Elazig (Turkey). She has published more than 30 articles in journals. Her research interests include analytical methods for nonlinear differential equations, numerical solutions of the partial differential equations and computer programming.

Hasan Bulut is currently a Prof. Dr. in Department of Mathematics at Firat University. He has published more than 100 articles in journals. His research interests include stochastic differential equations, fluid and heat mechanics, finite element method, analytical methods for nonlinear differential equations, mathematical physics, and numerical solutions of the partial differential equations, computer programming.

An International Journal of Optimization and Control: Theories \& Applications (http://ijocta.balikesir.edu.tr)

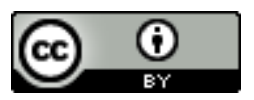

This work is licensed under a Creative Commons Attribution 4.0 International License. The authors retain ownership of the copyright for their article, but they allow anyone to download, reuse, reprint, modify, distribute, and/or copy articles in IJOCTA, so long as the original authors and source are credited. To see the complete license contents, please visit http://creativecommons.org/licenses/by/4.0/. 